\title{
Morfologia e produção de feijão comum em função da aplicação de bioestimulante
}

Morphology and common bean production due to the biostimulant

\author{
R. S. Silva ${ }^{1 *}$; J. J. N. L. Fogaça' ${ }^{1}$; E. S. Moreira ${ }^{1}$; T. R. Prado ${ }^{1}$; R. C. Vasconcelos ${ }^{2}$ \\ ${ }^{1}$ Programa de Pós-graduação em Agronomia - Fitotecnia, Universidade Estadual do Sudoeste da Bahia, 45031-900, \\ Vitória da Conquista, Brasil \\ ${ }^{2}$ Departamento de Fitotecnia e Zootecnia/Laboratório de grandes culturas, Universidade Estadual do Sudoeste da \\ Bahia, 45031-900, Vitória da Conquista, Brasil
}

*ricardo_deandrade@yahoo.com.br

(Recebido em 20 de maio de 2016; aceito em 30 de setembro de 2016)

\begin{abstract}
Com o objetivo de verificar os efeitos da aplicação do Biozyme $\mathrm{TF}^{\circledR}$ sobre as características agronômicas e componentes de produção do feijoeiro comum foi realizado um experimento na Universidade Estadual do Sudoeste da Bahia, em Vitória da Conquista. O delineamento utilizado foi o de blocos casualizados (DBC), quatro repetições e cindo tratamento, sendo uma testemunha e diferentes números de aplicações de Biozyme $\mathrm{TF}^{\circledR}$, perfazendo a mesma dose ao final do ciclo da cultura, $300 \mathrm{~mL}$ p.c. ha ${ }^{-1}$, uma aplicação aos 30 dias após a emergência da cultura, duas aplicações, aos 15 DAE e 30 dias após a emergência, três aplicações, aos 15, 30 e 45 DAE e quatro aplicações, aos 15, 30, 45 e 60 dias após a aplicação. Aos 65 dias após a emergência foi determinada a altura de plantas, diâmetro do caule, índice, número de folhas, área foliar e massa seca de parte aérea e aos 83 dias após a emergência, foram avaliados o comprimento de vagem, número de vagem por planta, massa de mil sementes, número de sementes por vagem e produtividade. Os dados foram submetidos à análise de variância e ao teste de Tukey a 5\%. A utilização do Biozyme $\mathrm{TF}^{\circledast}$ foi mais efetiva no incremento de massa quando aplicado na fase vegetativa e a aplicação deste bioestimulante não influenciam nos componentes de produção.
\end{abstract}

Palavras-chave: Phaseolus vulgaris, fitorreguladores, manejo de culturas

Insert the abstract here. In order to verify the effects of the application of Biozyme $\mathrm{TF}^{\circledR}$ on agronomic and common bean production components features an experiment was conducted at the State University of Southwest Bahia, Vitória da Conquista. The experimental design was a randomized block design (RBD), four replications-fifth treatment, being a witness and different numbers of Biozyme $\mathrm{TF}^{\circledR}$ applications,

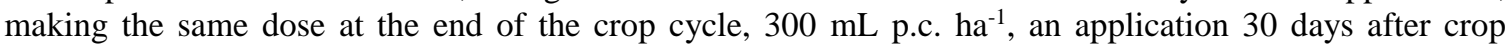
emergence, two applications at 15 DAE and 30 days after emergence, three applications at 15, 30 and 45 DAE and four applications at 15, 30, 45 and 60 days after application. After 65 days after emergence was determined the plant height, stem diameter, content, number of leaves, leaf area and dry weight of shoot and after 83 days after emergence, were evaluated the length of pod, pod number per plant, weight of thousand seeds, number of seeds per pod and productivity. The data were submitted to analysis of variance and 5\% Tukey test. The use of Biozyme $\mathrm{TF}^{\circledR}$ was more effective in the mass increase when applied in the vegetative stage and the application of biostimulant does not influence the production of components.

Keywords: Phaseolus vulgaris, fitorregulators, crop productions

\section{INTRODUÇÃO}

O Brasil destaca-se como um dos maiores produtores mundiais de feijão, com uma produção aproximada de 3,4 milhões de toneladas de grãos na safra 2013/2014 [5]. Este grão desempenha grande importância econômica e social, por ser componente básico da dieta alimentar e fonte de proteína para as classes sociais de menor renda.

Apesar de apresentar destaque na produção mundial, a produtividade brasileira é considerada baixa [2]. Assim, com intuito de elevar essa produtividade, novas tecnologias vêm sendo desenvolvidas. Kolling et al. [10] relatam que a utilização de bioestimulante é uma estratégia de manejo que favorece a expressão do potencial genético das plantas. 
Bioestimulantes são substâncias e/ou microrganismos que quando aplicados nas plantas as estimulam a aumentar a absorção e eficiência dos nutrientes e, consequentemente, na produtividade, fazem parte desse grupo: biorreguladores, aminoácidos, ácidos fúlvidos e húmicos e algas marinhas [8-13]. Estas atuam modificando ou alterando processos metabólicos e fisiológicos vegetais, como: aumento da divisão e alongamento celular; síntese de clorofila; fotossíntese; diferenciação das gemas florais; fixação e tamanho dos frutos; e absorção de nutrientes [7].

O Biozyme $\mathrm{TF}^{\circledR}$ é um bioestimulante composto por extratos vegetais, contendo três fitormônios biologicamente ativos: giberelina (ácido giberélico, $31 \mathrm{ppm}$ ), auxinas (ácido indolacético, $31 \mathrm{ppm}$ ) e citocininas (zeatina, $83 \mathrm{ppm}$ ), além de macro e micronutrientes, tais como enxofre $(0,44 \%)$; magnésio $(0,14 \%)$; boro $(0,30 \%)$; ferro $(0,49 \%)$; manganês $(0,12 \%)$ e zinco $(0,37 \%)$ [9]. Este produto é aplicado em plantas com a finalidade de estimular diferentes processos metabólicos e fisiológicos, como a diferenciação celular, translocação de substâncias, a síntese da clorofila, diferenciação broto, florescimento uniformização e pegamento de flores e frutos [16].

Manna et al. [12] estudando diferentes formulações e épocas de aplicações de Biozyme na cultura da pimenta observaram que plantas tratadas apresentaram adaptação às condições do ambiente e maior produtividade. Saul et al. [14] na cultura da goiaba verificaram que a aplicação do mesmo produto, na concentração de $10 \mathrm{ppm} \mathrm{pl}^{-1}$, aumentou a massa e o comprimento do sistema radicular, o número de frutos por planta, a produtividade média e os teores de clorofila A, B e total. No entanto, para a cultura do feijão há poucos relatos da eficiência do produto no aumento dos componentes de produção.

Diante do exposto, o objetivo deste trabalho foi verificar os efeitos da aplicação do Biozyme $\mathrm{TF}^{\circledR}$ sobre as características agronômicas e componentes de produção do feijoeiro comum (Phaseolus vulgaris).

\section{MATERIAL E MÉTODOS}

O estudo foi conduzido na Universidade Estadual do Sudoeste da Bahia (UESB), campus de Vitória da Conquista, situado a $14^{\circ} 51^{\prime} 58^{\prime \prime}$ latitude Sul e $40^{\circ} 50^{\prime} 22^{\prime}$ ' longitude Oeste, com altitude média de $923 \mathrm{~m}$. As médias de temperaturas máxima e mínima são, respectivamente, 23 e $15,8^{\circ} \mathrm{C}$, sendo o clima do tipo $\mathrm{C} 1 \mathrm{~dB}$ ' (subúmido a seco).

O solo da área experimental foi classificado como LVA distrófico, com textura média, com relevo suave ondulado e boa drenagem. A análise química do solo feita anterior à implantação do experimento, na profundidade de $0-20 \mathrm{~cm}$ de profundidade está apresentada na tabela 1 . $\mathrm{O}$ preparo do solo consistiu de uma aração e uma gradagem.

Tabela 1: Caracterização química do solo da área experimental, em março de 2015, na camada de 0 a 20 cm de profundidade, antes da implantação do experimento.

\begin{tabular}{|c|c|c|c|c|c|c|c|c|c|c|c|}
\hline$P$ & K & $\mathrm{Ca}$ & $\mathrm{Mg}$ & $\mathrm{Al}$ & $\mathrm{H}$ & S.B. & $\mathrm{T}$ & $\mathrm{T}$ & V & M & M.O. \\
\hline \multicolumn{2}{|c|}{$\mathrm{mg} \mathrm{dm} \mathrm{m}^{-3}$} & \multicolumn{7}{|c|}{ - } & \multicolumn{2}{|c|}{$\%$} & $\mathrm{mg} \mathrm{dm}{ }^{-3}$ \\
\hline 7 & 0,1 & 0,9 & 0,55 & 0,3 & 2,2 & 1,5 & 1,8 & 4,1 & 38 & 17 & 9 \\
\hline
\end{tabular}

O delineamento utilizado foi o de blocos casualizados (DBC), com cinco tratamentos e quatro repetições. As parcelas foram compostas por 4 linhas, espaçadas a $0,5 \mathrm{~m}$, de $5 \mathrm{~m}$ de comprimento, área total de $10,0 \mathrm{~m}^{2}$. Foram descartadas as duas linhas laterais e $0,5 \mathrm{~m}$ em cada extremidade, sendo a área útil final, de $4,0 \mathrm{~m}^{2}$.

Os tratamentos empregados no ensaio foram constituídos de diferentes números de aplicações do Bioestimulante Biozyme $\mathrm{TF}^{\circledR}$, perfazendo a mesma dose ao final do ciclo da cultura, $300 \mathrm{~mL}$ p.c. ha ${ }^{-1}$, dose recomendada pelo fabricante, e uma testemunha: $\mathrm{T} 1$ - testemunha, sem aplicação; T2 - uma aplicação de $300 \mathrm{~mL}$ p.c. ha $^{-1}$ aos 30 dias após a emergência da cultura (DAE); T3 duas aplicações de $150 \mathrm{~mL}$ p.c. ha- ${ }^{-1}$, aos 15 DAE e 30 DAE; T4 - três aplicações de $100 \mathrm{~mL}$ p.c. ha $^{-1}$, aos 15, 30 e 45 DAE; e T5 - quatro aplicações de $75 \mathrm{~mL}$ p.c. ha ${ }^{-1}$, aos 15, 30, 45 e 60 DAE. 
A variedade semeada foi a "carioquinha" do grupo carioca, a mais cultivada na região por considerada de alto potencial produtivo, o material foi adquirido com produtores locais. As sementes foram tratadas com metalaxil-m + fludioxonil $\left(300 \mathrm{~mL} 100 \mathrm{~kg}^{-1}\right)$. A semeadura foi realizada no dia 25 de abril de 2015, com adubação de base de $4000 \mathrm{~kg} \mathrm{ha}^{-1}$ do formulado 04-1408. Em adubação de cobertura, aos 15 e 30 dias após a emergência, utilizou-se $15 \mathrm{~kg} \mathrm{ha}^{-1}$ de ureia, $30 \mathrm{~kg} \mathrm{ha}^{-1}$ de cloreto de potássio e $0,5 \mathrm{~L} \mathrm{ha}^{-1}$ do complexo de nutrientes a base de $\mathrm{S}, \mathrm{Zn}, \mathrm{Mn}, \mathrm{B}$ e $\mathrm{Ca}$, nas concentrações de $5 \%, 0,5 \%, 2 \%, 1 \%$ e $4 \%$, respectivamente.

As aplicações dos tratamentos foram realizadas com pulverizador costal pressurizado a $\mathrm{CO}_{2}(2$ $\mathrm{kgf} \mathrm{cm}^{-2}$ ), acoplado a uma barra contendo dois bicos de jato leque plano $110.02 \mathrm{VS}$, com vazão de $200 \mathrm{~L} \mathrm{ha}^{-1}$. Durante a condução do experimento, foram realizados tratos culturais: como o controle de plantas daninhas, fitopatógenos e entomopatógenos, com capinas manuais, aplicação de herbicidas, e aplicação de inseticidas e fungicidas.

Aos 65 dias após a emergência foram feitas as seguintes determinações, em 10 plantas da área útil da parcela: Altura de plantas (ALT), medindo a distância do nível do solo à extremidade do ramo principal com régua graduada; Diâmetro do caule (DIAM), medido em $\mathrm{mm}$ por meio de um paquímetro digital; Índice SPAD (SPAD), medido em folhas completamente expandida do terço médio da planta, utilizando o clorofilômetro SPAD - 502; Número de folhas (NFOL), obtido pela média do NFOL; Área foliar (ARFOL), medida com integralizador de área modelo LI-310, Licor, Inglaterra; Massa seca de parte aérea (MSPA), as plantas foram secas em estufa a $65^{\circ} \mathrm{C}$ por 72 horas.

Aos 83 dias após a emergência, foram colhidas dez plantas por parcela para determinação do Comprimento de vagem (CV), média do comprimento de duas vagens localizadas no terço inferior da planta; Número de vagem por planta (NVP), média do número total de vagens; Massa de cem sementes (M100S), média em grama, de cem sementes, a média composta por oito medições por parcela; Número de sementes por vagem (NSV), determinado pela relação do número total de sementes das vagens das pelo número total de vagens. A produtividade (PROD) foi determinada pela colheita da área útil da parcela o resultado expresso em $\mathrm{kg} \mathrm{ha}^{-1}$, com umidade corrigida para $130 \mathrm{~g} \mathrm{~kg}^{-1}$.

Os dados foram submetidos à análise de variância (Teste F), e posteriormente, quando significativo aplicou-se o teste de Tukey a 5\% de probabilidade por meio do software Sisvar 5.1.

\section{RESULTADOS E DISCUSSÃO}

O parcelamento da aplicação do bioestimulante não influenciou na altura de plantas e no diâmetro do caule (Figura 1 A e B). Abrantes et al. [1] observaram resultados semelhantes para épocas de aplicação do biorregulador Stimulate ${ }^{\circledR}$, composto por auxina, giberelina e citocinina, os autores afirmam que as plantas do feijoeiro produzem hormônios suficientes para a orientação vegetativa, e que a aplicação exógena pode interferir na morfologia da planta, principalmente na fase reprodutiva, influenciando a altura de inserção da primeira vagem. No entanto, Manna et al. [12] verificaram que sob a aplicação de Biozyme $\mathrm{TF}^{\circledR}$, plantas de pimenta apresentam maiores alturas e características agronômicas de produção, porém a menor dose utilizada pelos autores foi dez vezes quatro vezes superior à dose final aplicada neste estudo. Assim, pode-se supor que, possivelmente, a dose utilizada não foi suficiente para interferir no processo produtivo da cultura do feijoeiro.

O Biozyme $\mathrm{TF}^{\circledR}$ além dos hormônios possui nutrientes essenciais para o desenvolvimento do aparato fotossintético, como nitrogênio e potássio solúvel, que resultou em maior número de folhas e área foliar em plantas que receberam a aplicação do regulador, exceto para o tratamento T4 (Figura $1 \mathrm{C}$ e D). O número de folhas e área foliar reduzida no tratamento T4 possivelmente ocorre, porque uma única aplicação do regulador, na fase inicial do período reprodutivo, interfira negativamente no balanço hormonal da planta. 

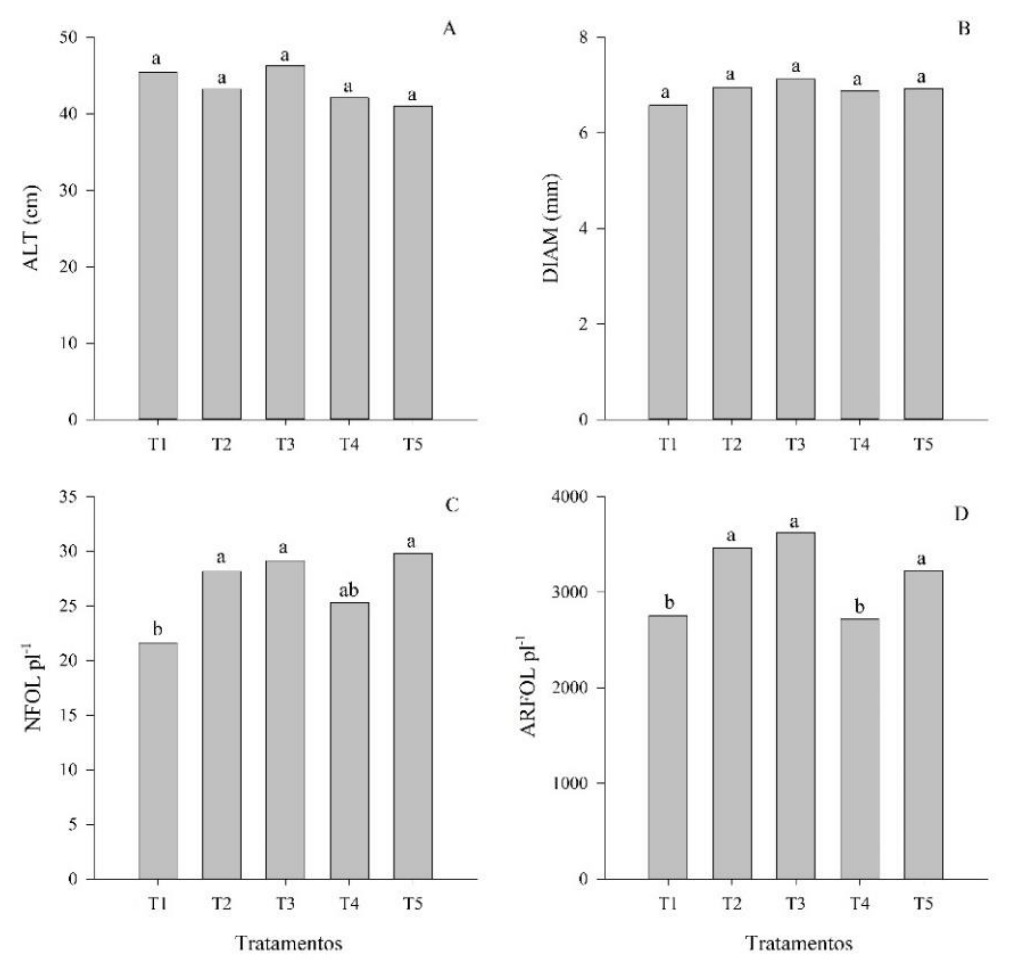

Figura 1: Média da altura de plantas $(A L T)(A)$, diâmetro do caule (DIAM) (B), número de folhas (NFOL) (C) e área foliar (ARFOL) (D), em resposta a diferentes números de aplicação do bioestimulante Biozyme $T F^{\circledR}$, na cultura do feijão, T2 - uma aplicação de $300 \mathrm{~mL}$ p.c. ha ${ }^{-1}$ aos 30 dias após a emergência da cultura (DAE); T3 - duas aplicações de $150 \mathrm{~mL}$ p.c. ha ${ }^{-1}$, aos 15 DAE e 30 DAE; T4 - três aplicações de $100 \mathrm{~mL}$ p.c. ha $\mathrm{s}^{-1}$, aos 15, 30 e 45 DAE; e T5 - quatro aplicações de $75 \mathrm{~mL}$ p.c. ha ${ }^{-1}$, aos 15, 30, 45 e 60

DAE, comparados à testemunha, sem aplicação.

Para a massa seca do caule, verificou-se que os tratamentos com uma e duas aplicações apresentaram maior massa que os demais tratamentos, e T5 maior massa que T4 (Figura 2C). Em relação à massa seca foliar, o tratamento com uma aplicação foi superior aos tratamentos sem aplicação e com três aplicações (Figura 2D). Analisando-se a massa seca total da parte aérea, dos tratamentos que receberam a aplicação do Biozyme $\mathrm{TF}^{\circledR}$, apenas o tratamento $\mathrm{T} 4$ foi igual a $\mathrm{T} 1$ (Figura 2A).

$\mathrm{O}$ fato das maiores massas terem sido observadas nos tratamentos que receberam aplicações apenas na fase vegetativa está relacionado com o estímulo para o crescimento vegetativo. Taiz e Zeiger [15] relatam que o ácido giberélico, a auxina e a citocinina, hormônios contidos no produto utilizado, são responsáveis pela divisão celular, promoção do crescimento, além de diferenciação de células meristemáticas, floema e xilema. 

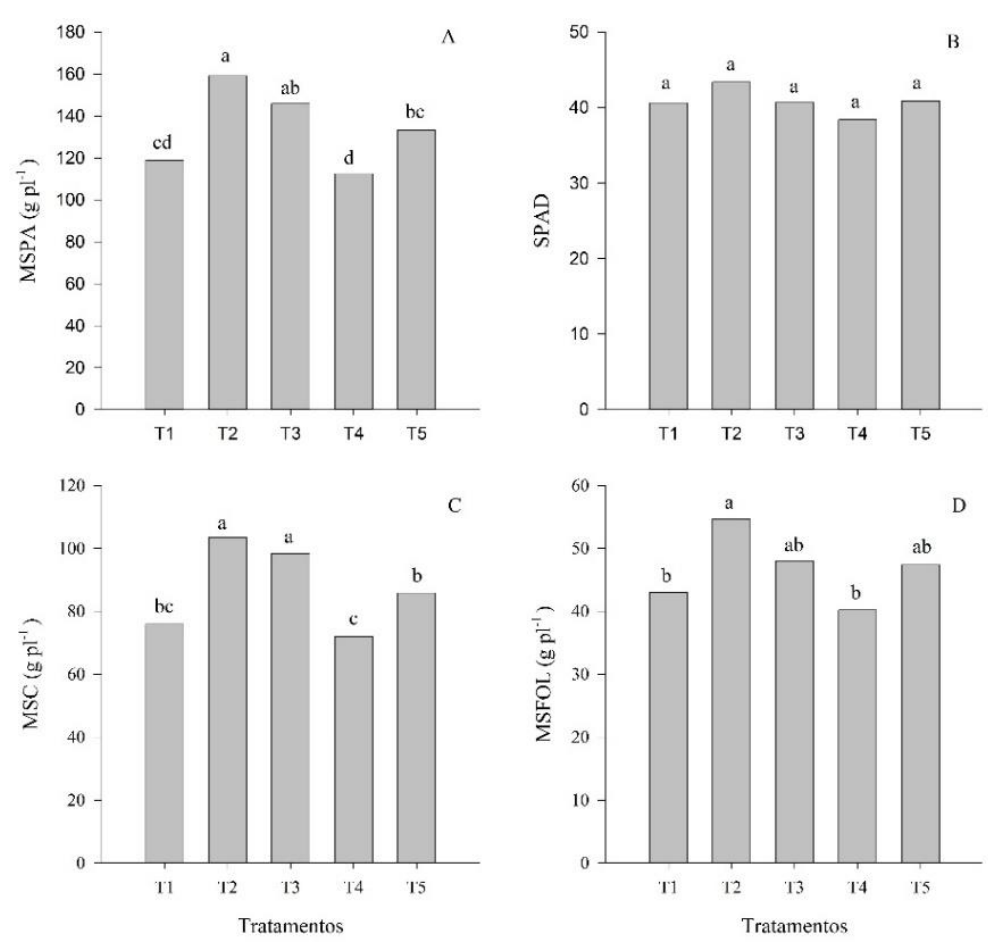

Figura 2: Média da massa seca de parte aérea (MSPA) (A), índice SPAD (B), massa seca de caule (MSC) (C) e massa seca de folhas $(M S F O L)(D)$, em resposta a diferentes números de aplicação do bioestimulante Biozyme $T F^{\circledR}$, na cultura do feijão, $T 2$ - uma aplicação de $300 \mathrm{~mL}$ p.c. ha ${ }^{-1}$ aos 30 dias após a emergência da cultura (DAE); T3 - duas aplicações de $150 \mathrm{~mL}$ p.c. ha-1 aos 15 DAE e 30 DAE; T4 - três aplicações de

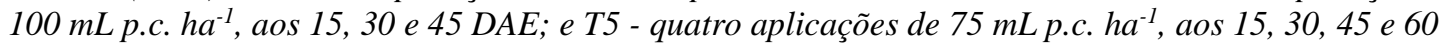
DAE, comparados à testemunha, sem aplicação.

A intensidade da coloração verde medida pelo índice SPAD não apresentou diferença entre os tratamentos estudados (Figura 2B), possivelmente a adubação nitrogenada de base e em cobertura foram suficientes para suprir a necessidade de nitrogênio para formação de clorofila, assim, mesmo na testemunha o índice SPAD foi considerado adequado para a cultura do feijoeiro.

Pela análise de variância, verificou-se que as variáveis, comprimento de vagens, número de vagens por planta, número de grãos por vagem, massa de cem sementes, índice de grãos por vagem e produtividade não apresentaram diferença significativa entre os tratamentos (Tabela 2). Esses resultados concordam com os apresentados por Dourado Neto et al. [6], que trabalhando com aplicação do fitorregulador na cultura do feijão, observaram que devido ao efeito dos bioestimulantes ser variável em função do estádio fenológico da planta, sua ação é mais efetiva na emergência das plântulas e no desenvolvimento inicial.

Tabela 2: Média do comprimento de vagens (CVA), número de vagens por planta (NVGP), massa de cem sementes (M100S), número de grãos por vagem (NVG), índice de grãos por vagem (IGRAOS) $e$ produtividade (PROD), em resposta a diferentes números de aplicação do bioestimulante Biozyme $T F^{\circledR}, n a$ cultura do feijão, comparados a testemunha.

\begin{tabular}{ccccccc}
\hline Tratamentos & CVA & NVGP & M100S & NGV & IGRAOS & PROD \\
\hline T1 & $11,11 \mathrm{a}$ & $15,30 \mathrm{a}$ & $27,66 \mathrm{a}$ & $5,65 \mathrm{a}$ & $0,75 \mathrm{a}$ & $3190,00 \mathrm{a}$ \\
T2 & $11,35 \mathrm{a}$ & $14,30 \mathrm{a}$ & $26,83 \mathrm{a}$ & $5,85 \mathrm{a}$ & $0,74 \mathrm{a}$ & $3233,33 \mathrm{a}$ \\
T3 & $12,00 \mathrm{a}$ & $17,45 \mathrm{a}$ & $26,83 \mathrm{a}$ & $5,81 \mathrm{a}$ & $0,75 \mathrm{a}$ & $3347,22 \mathrm{a}$ \\
T4 & $11,92 \mathrm{a}$ & $15,60 \mathrm{a}$ & $27,28 \mathrm{a}$ & $8,77 \mathrm{a}$ & $0,78 \mathrm{a}$ & $3266,66 \mathrm{a}$ \\
T5 & $11,81 \mathrm{a}$ & $17,02 \mathrm{a}$ & $27,25 \mathrm{a}$ & $6,17 \mathrm{a}$ & $0,76 \mathrm{a}$ & $3383,33 \mathrm{a}$ \\
\hline CV & 4,86 & 13,54 & 3,67 & 9,73 & 3,49 & 11,48 \\
\hline
\end{tabular}

As médias seguidas pela mesma letra na coluna não diferem entre si pelo Teste de Tukey ao nível de 5\% de probabilidade. Sendo T1 - testemunha, sem aplicação; T2 - uma aplicação de $300 \mathrm{~mL}$ p.c. ha ${ }^{-1}$ aos 30 dias após a emergência da cultura (DAE); T3 - duas aplicações de $150 \mathrm{~mL}$ p.c. ha ${ }^{-1}$, aos 15 DAE e 30 DAE; T4 - três aplicações de $100 \mathrm{~mL}$ p.c. ha ${ }^{-1}$, aos 15, 30 e 45 DAE; e T5 - quatro aplicações de $75 \mathrm{~mL}$ p.c. ha- ${ }^{-1}$, aos 15, 30, 45 e 60 DAE. 
Mesmo não apresentando diferenças estatísticas significativas entre os componentes de produção, quando comparados todos os tratamentos, é importante frisar que, quando aplicado o Biozyme $\mathrm{TF}^{\circledR}$ em 2 e 4 aplicações, verificou-se um acréscimo de 157 e $193 \mathrm{~kg} \mathrm{ha}^{-1}$, incremento de 4,9 e $6,0 \%$ na produtividade da cultura, respectivamente. Estudos complementares para validação do uso desse produto na elevação da produção devem ser realizados, buscando novos parâmetros fitotécnicos de recomendação.

A utilização eficiente de insumos agrícolas é algo preponderante para minimizar os custos de produção e elevar os patamares produtivos, porém quanto maior o nível tecnológico implantado na lavoura, mais difícil é a obtenção de incrementos de produtividade pela adoção de novas práticas de manejo. Apesar de bem difundidos, faltam resultados sólidos sobre a eficiência dos bioestimulantes, principalmente, relacionados ao aumento de produtividade em diversas culturas, isso devido os mecanismos de ação dos bioestimulantes não serem completamente entendidos, muitas vezes esses produtos atuam modulando respostas a estresses abióticos em plantas, podendo ou não resultar em aumento de produção [4].

Os resultados de experimentos realizados com uso de bioestimulantes em culturas anuais como, milho, soja, feijão e algodão apresentam-se inconsistentes, a aplicação dessas substâncias visa promover o equilíbrio hormonal das plantas, no entanto, nem sempre há respostas que viabilizem a adoção dessa prática de forma sistêmica. Dourado Neto et al. [6] e Lana et al. [4] concluíram que a aplicação dos bioestimulantes testados elevou a produção de grãos de feijão em relação à testemunha. Enquanto que Anjos et al. [3] não verificaram incremento em produtividade após a aplicação de biorreguladores na cultura do feijão e da soja, respectivamente.

\section{CONCLUSÃO}

A utilização do Biozyme $\mathrm{TF}^{\circledR}$ é mais efetiva no incremento de massa na fase vegetativa de feijoeiro comum variedade carioquinha e não influencia nos componentes de produção.

\section{REFERÊNCIAS BIBLIOGRÁFICAS}

1. Abrantes FL, Sá ME, Souza LCD, Silva MP, Simidu HM, Andreotti M, Buzetti S, Valério Filho WV, Arruda N. Uso de regulador de crescimento em cultivares de feijão de inverno. Pesquisa Agropecuária Tropical, 2011; 41(2):148-154, doi: 10.5216/pat.v41i2.8287.

2. Amaro HTR, David AMS, Carvalho AJ, Aspiazú I, Silva Neta IC, Santos MGP. Physiological potential of bean seeds as a function of population densities on winter crop season, in northern Minas Gerais. Bioscience Journal, Uberlandia, 2015; 31(2): 415-424.

3. Anjos DN, Vasconcelos RC, Mendes HTA, Alcantara ASA, Viana AES. Biostimulants, macro and micronutrient fertilizer influence on common bean crop in Vitória da Conquista - Ba, Brazil. African Journal of Agricultural Research, 2015; 10(16): 1891-1897, doi: 10.5897/AJAR2014.9359.

4. Calvo P, Nelson L, Kloepper JW. Agricultural uses of plant biostimulants. Plant and Soil, 2014; 383(1): 3-41, doi: 10.1007/s11104-014-2131-8.

5. CONAB - Companhia Nacional de Abastecimento. Indicadores da Agropecuária. Revista Observatório Agrícola, Ano XXIV, 2015; 2(1): 18.

6. Dourado Neto D, Dario GJA, Barbieri APP, Martin TN. Ação de bioestimulante no desempenho agronômico de milho e feijão. Bioscience Journal, Uberlandia, 2014; 30(1): 371-379.

7. Du Jardin P. Plant biostimulants: Definition, concept, main categories and regulation. Scientia Horticulturae, 2015; 196(1): 3-14, doi:10.1016/j.scienta.2015.09.021.

8. Gómez-Merino FC, Trejo-Téllez LI. Biostimulant activity of phosphite in horticulturae. Scientia Horticulturae, 2015; 196(1): 82-90, doi:10.1016/j.scienta.2015.09.035.

9. González-Cortés N, Jiménez VR, Guerra BEC, Silos EH, De la Cruz PE. Germinación del chile amashito (Capsicum annuum L. var. Glabriusculum) en el sureste mexicano. Revista Mexicana de Ciencias Agrícolas, 2015; 196(1): 2211-2218, doi:10.1016/j.scienta.2015.09.035.

10. Kolling DF, Sangoi L, Souza CA, Schenatto DE, Giordani W, Boniatti CM. Tratamento de sementes com bioestimulante ao milho submetido a diferentes variabilidades na distribuição espacial das plantas. Ciência Rural, Santa Maria, 2016; 46(2):248-253, doi.org/10.1590/0103-8478cr20150504. 
11. Lana AMQ, Lana RMQ, Gozuen CF, Bonotto I, Trevisan LR. Aplicação de reguladores de crescimento na cultura do feijoeiro. Bioscience Journal, 2009; 25(1):13-20.

12. Manna D, Sakar A, Mayti TK. Impact of biozyme on growth, yeld and quality of chilli [Capsicum annum L]. Journal of Crop and Weed, 2012; 8(1): 40-43.

13. Nardi S, Pizzeghello D, Schiavon M, Ertani A. Plant biostimulants: physiological responses induced by preotein hydrolyzed-based products and humic substances in plant metabolism. Scientia Agricola, 2016; 73(1):18-23, doi.org/10.1590/0103-9016-2015-0006.

14. Sau1 S, Ghosh B, Sarkar S, Deb P. Effect of foliar application of biozyme on yield and physicochemical properties of rainy season crop of guava (Psidium guajava L.) cv. Allahabad safeda in alluvial soil of west Bengal. International Journal of Bio-Resource, Environment and Agricultural Sciences, 2015; 1(4): 176-185.

15. Taiz L, Zeiger E. Fisiologia vegetal, $5^{\mathrm{a}}$ ed, Trad, de E, R, Santarém, Porto Alegre: Artmed, 2013.

16. Tandon S, Dubey A. Effect of Biozymetm (Ascophyllum Nodosum) Biostimulant on Growth and Development of Soybean [Glycine $\operatorname{Max} \quad$ (L.) Merill]. 2015; 21:22, doi: 10.1080/00103624.2015.1011749. 